\title{
Synthesis and biological evaluation of substituted naphthoquinone derivatives as potent antimycobacterial agents
}

\author{
A. Mital, ${ }^{a, *}$ V.S. Negi, ${ }^{a}$ and U. Ramachandran ${ }^{b}$ \\ ${ }^{a}$ Department of Pharmaceutical Technology, National Institute of Pharmaceutical Education and \\ Research, Sector 67, Phase X, S. A. S. Nagar 160062, India \\ ${ }^{b}$ Orchid Chemicals \& Pharmaceuticals Ltd., 476/14, Old Mahabalipuram Road, Sholinganallur
} Chennai 600 119, India

Email: alkamital@gmail.com

\begin{abstract}
In the present study, a series of 1,4-naphthoquinone derivatives were synthesized and screened for antitubercular activity against Mycobacterium tuberculosis $\mathrm{H}_{37} \mathrm{Rv}$ strain by the broth microdilution assay method. The in vitro antitubercular activity data of the tested compounds against $M$. tuberculosis strain $\mathrm{H}_{37} \mathrm{Rv}$ showed moderate to good activity against bacteria compared to reference drugs. The most effective compounds of the series are 26, 28, 29, 34 and 36 with $\mathrm{IC}_{50}$ values ranging from $3.9-0.3 \mu \mathrm{g} / \mathrm{mL}$. The objective of our study is to generate new leads that operate through a different mode of action and to optimize their structure to display potent efficacy.
\end{abstract}

Keywords: Tuberculosis, Mycobacterium tuberculosis, Antimycobacterial activity, Naphthalene-1,4-diones

\section{Introduction}

Tuberculosis is the greatest single infectious cause of mortality worldwide. It is estimated that about one-third of the world's population is infected with this disease. ${ }^{1}$ Around 3 million people die annually from tuberculosis and there are about 9 million new cases each year, ${ }^{2-4}$ out of which the major share occur in developing countries. The highly effective combined therapy regimen of isoniazid (INH), rifampicin (RIF), and pyrazinamide (PZA) forms the basis of the current DOTS (directly observed therapy, short course), which is vigorously implemented. These three drugs with significantly different chemical structures affect different essential metabolic pathways of M. tuberculosis. The emergence of multi-drug resistant strains of M. tuberculosis, even in the course of successful DOTS programs, emphasizes the need for new chemical entities with novel mechanisms of action. ${ }^{5,6}$ Furthermore, treatment of this disease caused by resistant bacteria is 
more difficult in patients infected with human immunodeficiency virus (HIV). ${ }^{7}$ Because of this, there is an urgent need for anti-TB drugs with improved properties such as enhanced activity against MDR strains, reduced toxicity and shortened duration of therapy.

1,4-Naphthoquinone structure is common in various natural products ${ }^{8}$ and is found to exhibit an interesting range of pharmacological properties including antibacterial, ${ }^{9,10}$ antiviral, ${ }^{11}$ trypanocidal, ${ }^{12}$ anticancer, ${ }^{13}$ antimalarial ${ }^{14,15}$ and antifungal ${ }^{16}$ activities. Other quinone-related scaffolds such as 2- $H$-pyran-3(6H)-one derivatives ${ }^{17}$ and 2,3-dideoxyhexenopyranosides ${ }^{18}$ are also known for their biological activity against Gram positive bacteria such as Staphylococcus aureus and M. tuberculosis. South African medicinal plants have been traditionally used in the treatment of chest complaints. The significant activity of the crude extracts of Euclea natalensis ${ }^{19}$ and that of diospyrin 1 Fig. (1), isolated from this plant was observed against drugresistant strains of $M$. tuberculosis. ${ }^{20}$ It has been shown that a 2 -aminoacetate derivative of dimethylether-diospyrin has enhanced antituberculosis activity. ${ }^{21}$ The monomer of diospyrin $\mathbf{1}$, 7-methyljuglone 2 Fig. (1), exhibited intracellular and extracellular inhibition of M. tuberculosis comparable to streptomycin and ethambutol. ${ }^{22}$

A combination treatment of diospyrin $\mathbf{1}$ and methyljuglone $\mathbf{2}$, which may be more effective than a single drug treatment of the two naphthoquinones, is also being considered. The biological activity of naphthalene-1,4-diones is mainly due to the presence of two carbonyl groups that have the ability to accept one and/or two electrons to form the corresponding radical anion or dianion species as well as their acid-base properties. ${ }^{23}$ There are no published reports examining the toxicity of quinones against the genus Mycobacterium.

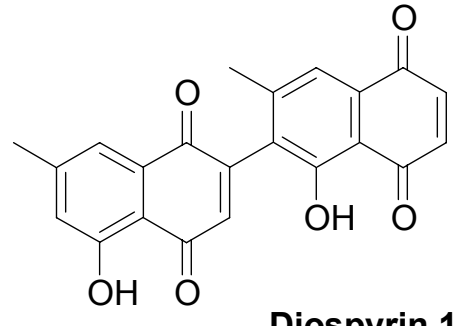

Diospyrin 1<smiles>Cc1cc(O)c2c(c1)C(=O)C=CC2=O</smiles>

7-methyljuglone 2

Figure 1. Structures of naphthoquinones.

We have been involved in the design, synthesis and bioevaluation of new antitubercular agents which are structurally different from known anti-tuberculosis drugs, and several naphthoquinone derivatives 3, 4, 5 and the aminoquinoline derivative 6 Fig. (2) with antitubercular activity have been reported earlier. ${ }^{24,25}$ 
<smiles>CC(=O)c1ccnc(C2=CC(=O)c3ccccc3C2=O)c1</smiles>

3<smiles>Cc1ccc(C2=CC(=O)c3ccccc3C2=O)nc1</smiles>

4<smiles>CC(=O)OC(C)=O</smiles><smiles>FC(F)(F)c1cc(NCCN2CCCCC2)c2cccc(C(F)(F)F)c2n1</smiles>

Figure 2. Naphthoquinone and aminoquinoline derivatives.

Compound 3 (Fig. 2) was considered as a lead molecule in our previous paper ${ }^{25}$ and subsequent structural modifications were carried out to synthesize different analogs, wherein most of the alterations made have shown promising activities. In continuation of our search, this second communication describes the synthesis and primary screening of 2-substituted naphthalene-1,4-dione derivatives against $M$. tuberculosis strain H37Rv. These derivatives 15-41 were synthesized following standard procedures starting from either naphthalene-1,4-dione 7 or 2-bromonaphthalene $\mathbf{8}^{26}$ The oxidative coupling of naphthalene-1,4-dione 7 with 2-substituted heterocycles in acetic acid and stoichiometric amounts of palladium acetate under reflux conditions gave the corresponding 2-heteroaryl-substituted naphthalene-1,4-diones 15-20, 29, 30, 31, and 33-41 (Scheme 1). Compounds 21-24 and 32 were prepared via Heck coupling of 2bromonaphthalene $\mathbf{8}$ with different acrylic esters to give 2-substituted naphthalene esters 9-13 and followed by their oxidation with periodic acid and chromium trioxide (Scheme 2). Derivatives 25-28 were prepared by condensation of 1,4-naphthoquinone 7 with 3-mercapto propionic acid $\mathbf{1 4}$ at room temperature, followed by the treatment of the acid derivative 25 with thionyl chloride and the appropriate amine (Scheme 3). All new compounds were fully characterized on the basis of spectroscopic and analytical data. 


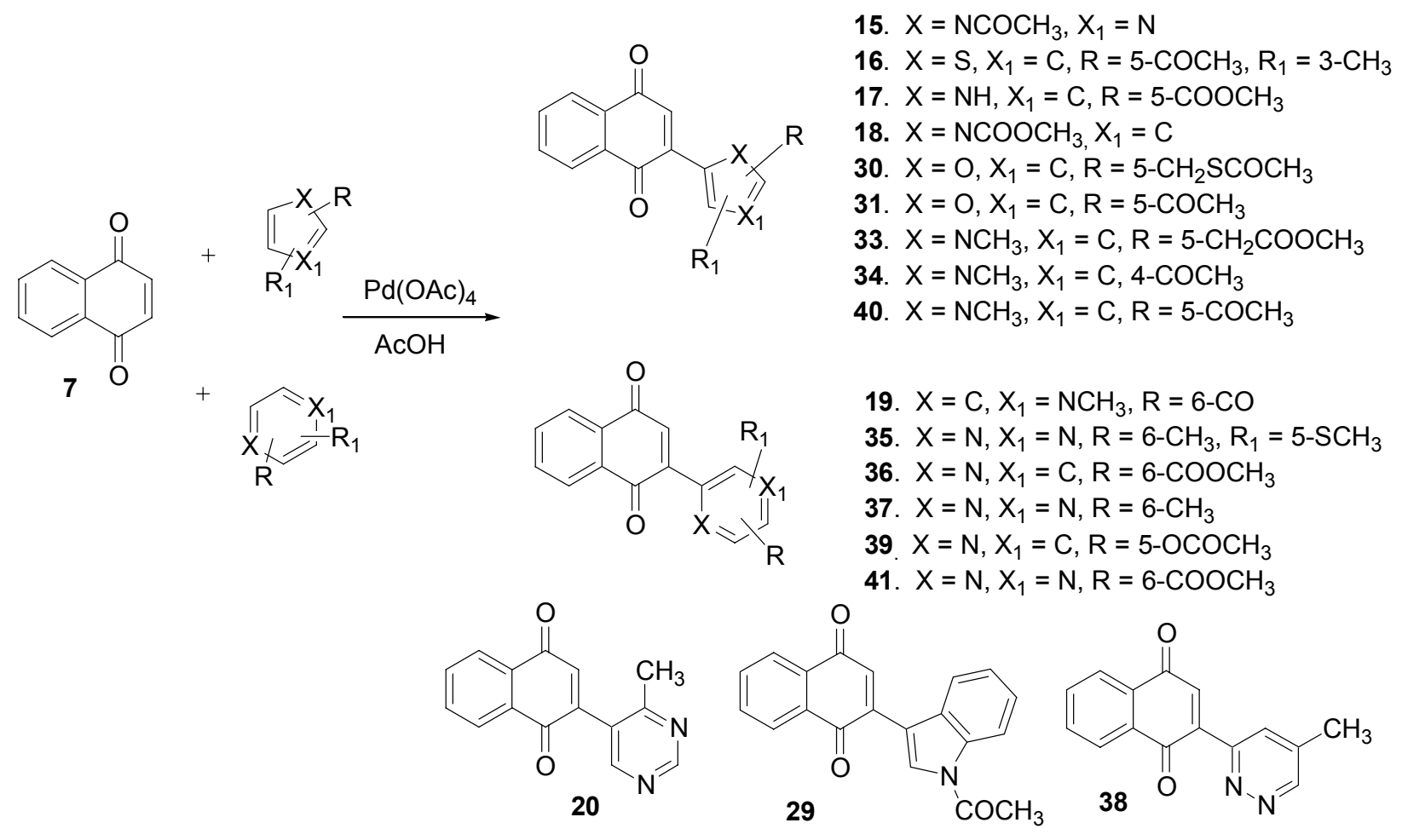

Scheme 1. Oxidative coupling of naphthalene-1,4-dione with 2-substituted heterocycles.

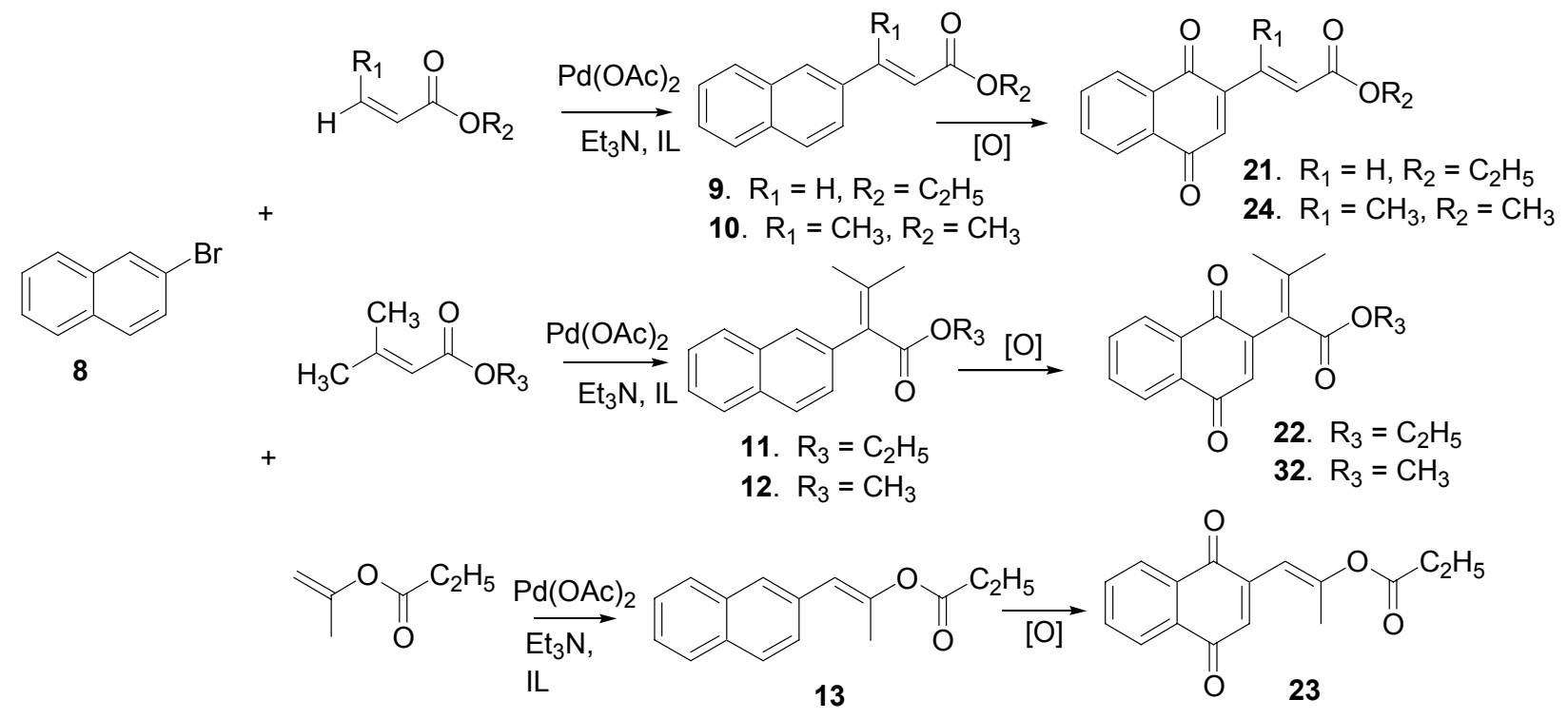

Scheme 2. Heck coupling of 2-bromonaphthalene with various vinyl esters and oxidation. 


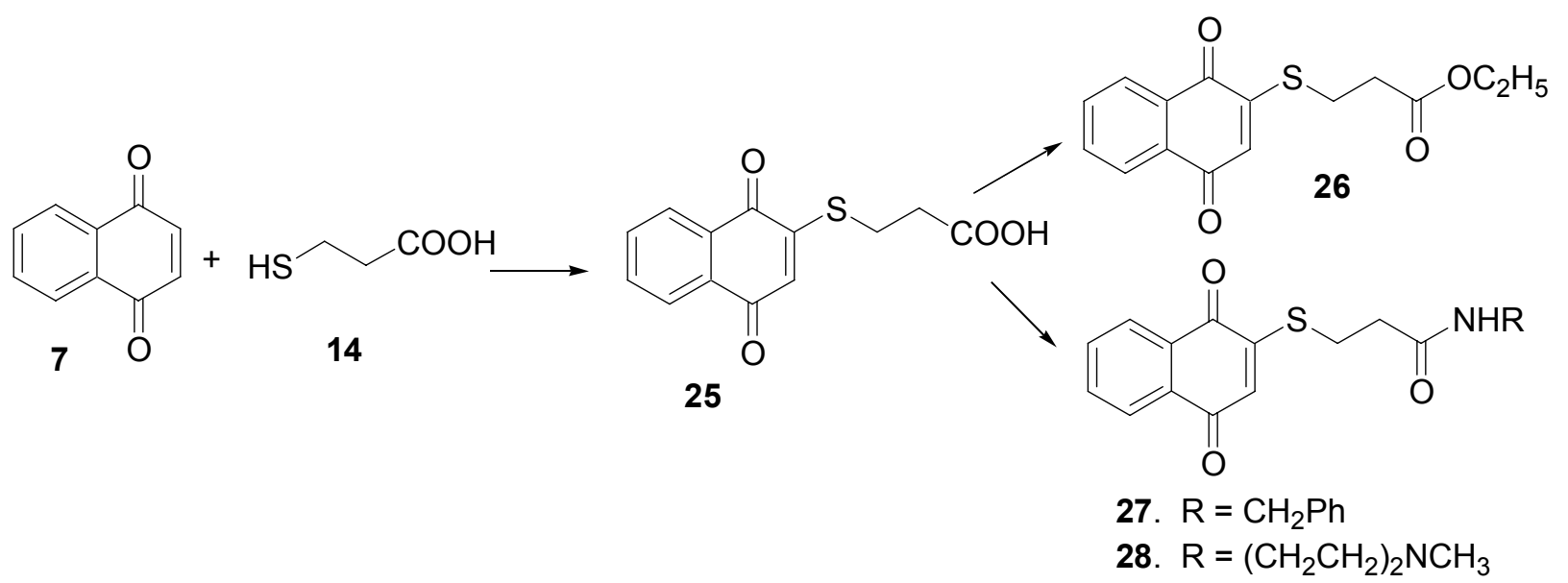

Scheme 3. Coupling of naphthalene-1,4-dione with 3-mercaptopropionic acid and amines.

\section{Results and Discussion}

All the synthesized compounds were primarily screened against M. tuberculosis strain $\mathrm{H}_{37} \mathrm{Rv}$ (ATCC 27294) using the microplate alamar blue assay (MABA). ${ }^{27,28}$ The results of the in vitro evaluation of antituberculosis activity are summarized in Table 1. During the preliminary screening six compounds $\left(\mathbf{2 6}, \mathbf{2 8}, \mathbf{2 9}, 34\right.$ and 36) were active and have $\mathrm{IC}_{50}$ values ranging from $3.974-0.319 \mu \mathrm{g} / \mathrm{mL}$, while all others were weakly active. Compounds 15, 16, 21 and 22 also showed $96.68 \%, 100 \%, 97.9 \%$ and $99.94 \%$ growth inhibitions, respectively at $6.25 \mu \mathrm{g} / \mathrm{mL}$. Compound $\mathbf{3 4}$ was further screened by serial dilution to assess cytotoxicity to a VERO cell line, to determine the selectivity index (SI), defined as the ratio of the measured $\mathrm{IC}_{50}$ in VERO cells to the MIC described above. Compound 34 showed good in vitro profile with $\mathrm{IC}_{50}$ value of 3.97 $\mu \mathrm{g} / \mathrm{mL}$ and $\mathrm{EC}_{50}$ of $10.89 \mu \mathrm{g} / \mathrm{mL}$, but the corresponding SI (2.16) against M.tb $\mathrm{H}_{37} \mathrm{Rv}$ strain is too low to be considered significant.

The naphthoquinone derivatives discovered in this study may provide valuable therapeutic intervention for the treatment of antitubercular diseases. It is conceivable that those naphthoquinone derivatives bearing heterocyclic rings might act as leads for optimizing the antitubercular activity. It will be interesting to prepare new analogues of the most active compounds having either heterocyclic rings or open chains which may be nontoxic and with significant antitubercular activity. 
Table 1. In vitro antimycobacterial activities and in silico parameters of naphthalene-1,4-dione derivatives, expressed as IC values $(\mu \mathrm{g} / \mathrm{mL})$ against drug-sensitive strain of $M . t b \mathrm{H}_{37} \mathrm{Rv}$

\begin{tabular}{|c|c|c|c|c|c|c|}
\hline S.No. & Structure & $\begin{array}{l}\text { Mol. } \\
\text { Wt. }\end{array}$ & $\begin{array}{l}\text { No. of } \\
\text { rotatable } \\
\text { bonds }\end{array}$ & $\begin{array}{l}\text { PSA } \\
\left(\AA^{2}\right)\end{array}$ & $\begin{array}{c}\operatorname{LogP} \text { at } \\
\text { pH } 7.4\end{array}$ & $\begin{array}{c}\text { IC90 \& } \\
50 \\
(\mu \mathrm{g} / \mathrm{ml})\end{array}$ \\
\hline 15 & & 266 & 2 & 66.81 & 0.69 & $\begin{array}{r}27.688, \\
18.633\end{array}$ \\
\hline 16 & & 296 & 2 & 51.21 & 3.21 & $\begin{array}{l}15.92 \\
11.384\end{array}$ \\
\hline 17 & & 281 & 3 & 72.47 & 2.31 & $\begin{array}{l}>100 \\
>100\end{array}$ \\
\hline 18 & & 281 & 3 & 63.68 & 2.16 & $\begin{array}{l}>100 \\
>100\end{array}$ \\
\hline 19 & & 265 & 1 & 54.45 & 1.37 & $\begin{array}{l}45.765, \\
34.231\end{array}$ \\
\hline 20 & & 250 & 1 & 59.92 & 1.46 & $\begin{array}{c}38.453 \\
32.272\end{array}$ \\
\hline
\end{tabular}


Table 1. Continued

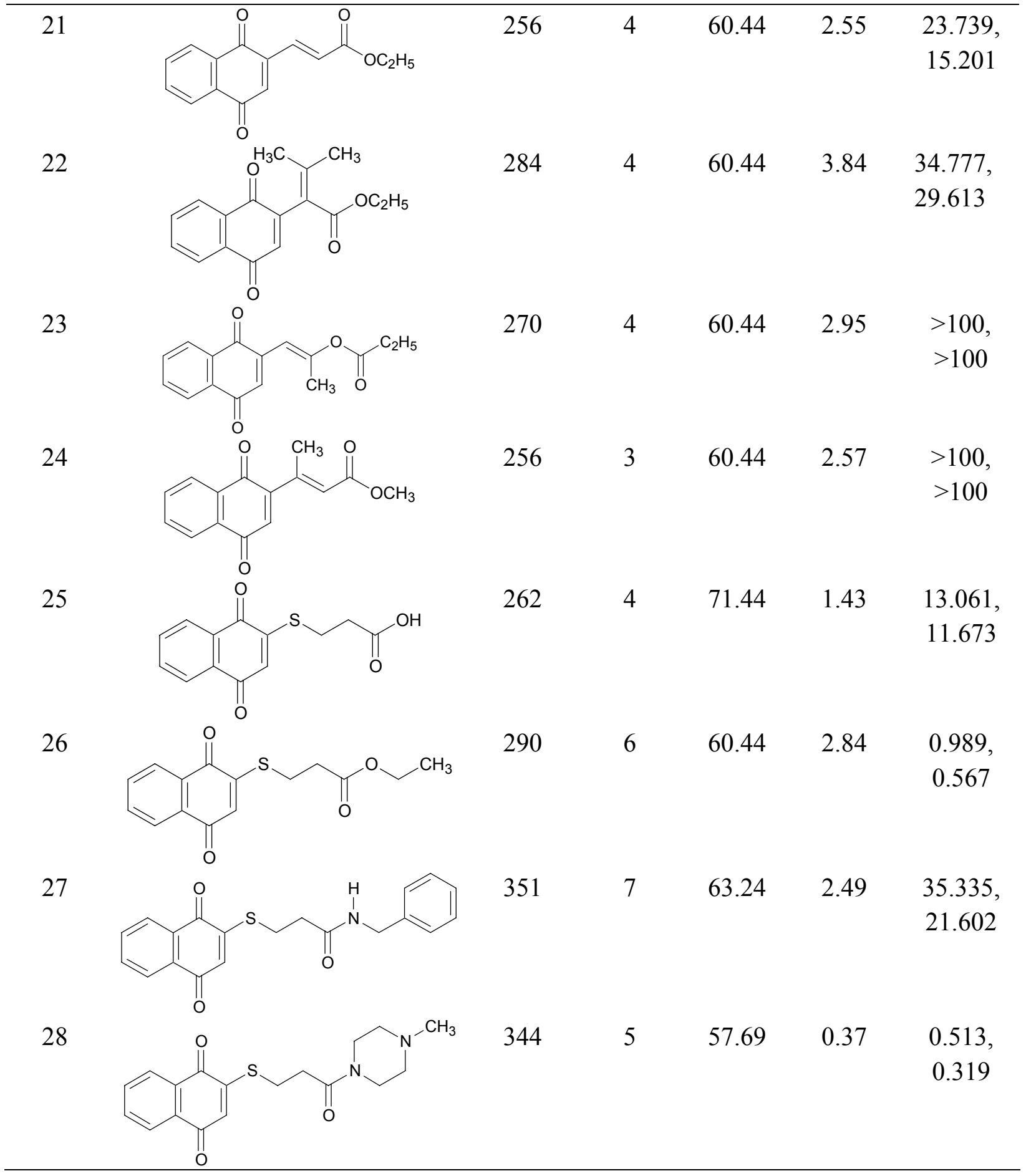


Table 1. Continued

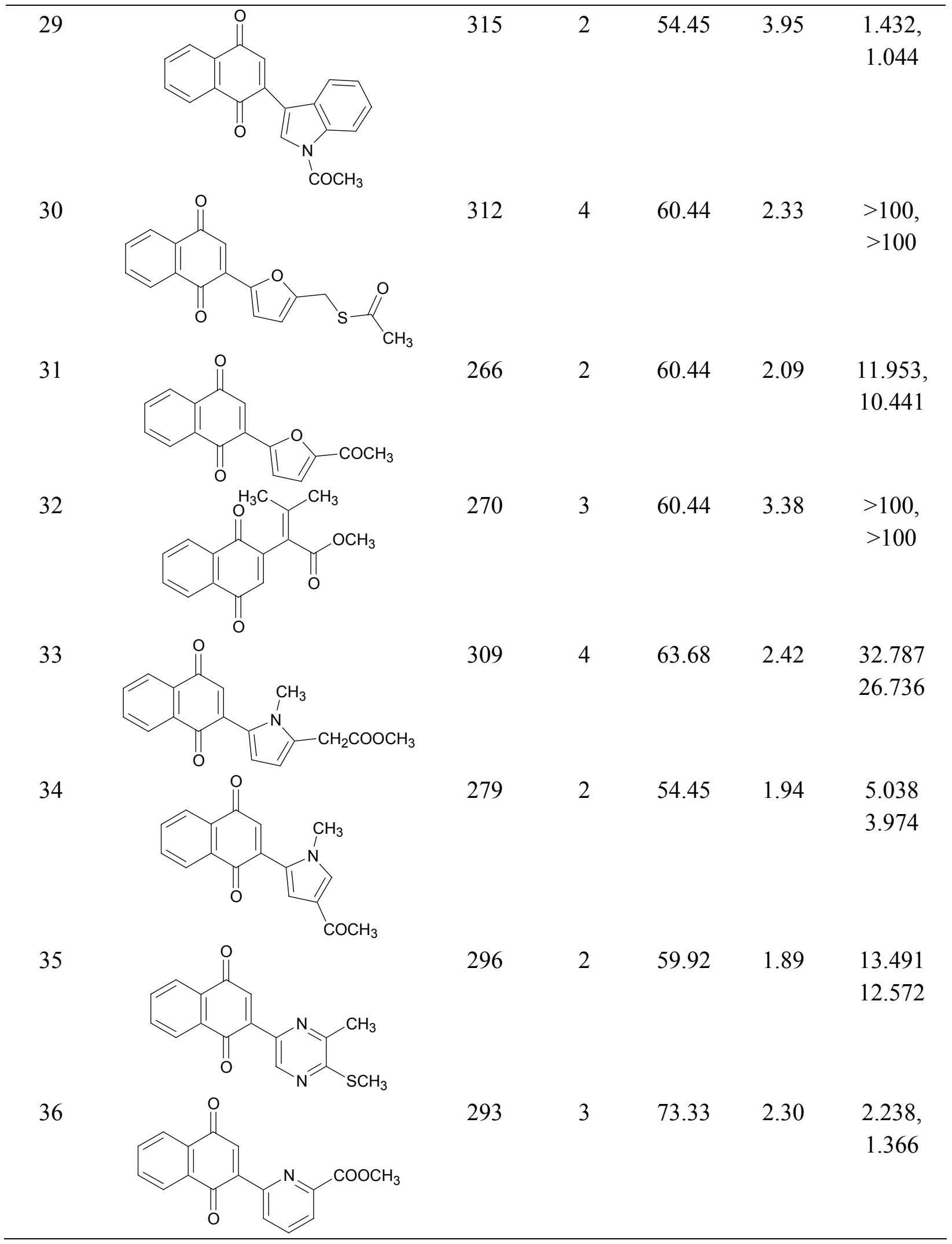


Table 1. Continued

\begin{tabular}{|c|c|c|c|c|c|}
\hline 37 & 250 & 1 & 59.92 & 1.20 & $\begin{array}{l}25.517 \\
22.462\end{array}$ \\
\hline 38 & 250 & 1 & 59.92 & 1.82 & $\begin{array}{c}11.934 \\
9.604\end{array}$ \\
\hline 39 & 293 & 3 & 73.33 & 1.79 & $\begin{array}{c}18.943 \\
13.286\end{array}$ \\
\hline 40 & 279 & 2 & 54.45 & 2.00 & $\begin{array}{l}>100 \\
>100\end{array}$ \\
\hline 41 & 294 & 3 & 86.22 & 0.66 & $\begin{array}{c}6.588 \\
5.998\end{array}$ \\
\hline
\end{tabular}

\section{Experimental Section}

General Procedures. Melting points were recorded on a Büchi capillary melting point apparatus and are uncorrected. Infrared (IR) spectra were recorded on a Nicolet Impact-410 FTIR spectrometer. ${ }^{1} \mathrm{H}$ spectra were recorded on a $300 \mathrm{MHz}$ Bruker FT-NMR spectrometer in $\mathrm{CDCl}_{3}$ solution. The chemical shifts are reported in $\delta(\mathrm{ppm})$ relative to internal standard tetramethylsilane (TMS) and coupling constants $J$ are given in Hz. Mass spectrometry was conducted using GCMS (Shimadzu QP 5000 spectrometer) auto sampler/direct injection (EI/CI) or LCMS (Finnigan Mat LCQ spectrometer) (APCI/ESI). Elemental analyses were recorded on an Elementar Vario EL analyzer. All chromatographic purifications were performed with silica gel 60 (230-400 mesh), whereas all TLC development was done on silica gel coated (Merck Kiesel 60 F254, $0.2 \mathrm{~mm}$ thickness) plates. All chemicals were purchased from Aldrich Chemical Company (USA) and were used as received unless otherwise noted. Solvents used for the 
chemical synthesis were of laboratory and analytical grade, and were used without further purification unless otherwise stated.

\section{General method for the synthesis of derivatives 15-20, 29, 30, 31 and 33-41 (Scheme 1)}

A solution of naphthalene-1,4-dione 7, the substituted aromatic heterocyclic compounds and palladium acetate in glacial acetic acid in the ratios 1:1:1 were heated at reflux temperature under a nitrogen atmosphere for 10-24 hours. The reaction mixture was evaporated to give a residue, which was then chromatographed on a silica gel column and eluted with hexane: ethyl acetate to give the corresponding 2-substituted naphthoquinone derivatives 15-20, 29, 30, 31 and 33-41.

\section{General method for the synthesis of derivatives 21-24, and 32 (Scheme 2)}

The ionic liquid [bmim]PF6 (0.445 g, $1.4 \mathrm{mmol})$, palladium acetate $(0.027 \mathrm{~g}, 0.12 \mathrm{mmol})$, and tris- $o$-tolylphosphine $(0.073 \mathrm{~g}, 0.24 \mathrm{mmol})$ were mixed in a three-necked round bottom flask and heated to $120{ }^{\circ} \mathrm{C}$ under a nitrogen atmosphere for 10-15 minutes till a yellowish brown solution of catalyst in the ionic liquid was obtained. Acrylic ester $(2.90 \mathrm{mmol})$, triethylamine $(0.366 \mathrm{~g}$, $3.63 \mathrm{mmol})$ and 2-bromonaphthalene $8(0.500 \mathrm{~g}, 2.42 \mathrm{mmol})$ were added to the solution and the reaction mixture was heated at $70{ }^{\circ} \mathrm{C}$ for 4 hours. After the completion of the reaction (monitored by TLC), $n$-hexane $(25 \mathrm{ml})$ was added to the reaction mixture and extraction was done for 15 minutes with vigorous stirring. The extraction was repeated with $n$-hexane $(2 \times 25 \mathrm{ml})$. The organic extracts were combined and concentrated under reduced pressure to give the crude product, which was purified by silica gel column chromatography.

\section{General procedure for the oxidation of 2-substituted naphthalene ester derivatives}

To an ice-cooled solution of periodic acid $(0.957 \mathrm{~g}, 4.2 \mathrm{mmol})$ and chromium trioxide $(0.015 \mathrm{~g}$, $0.1 \mathrm{mmol})$ in acetonitrile $(25 \mathrm{ml})$ was added all at once the 2-substituted naphthalene ester derivative 9-13 (1 mmol). A white precipitate was formed immediately in an exothermic reaction. After stirring the reaction at $5{ }^{\circ} \mathrm{C}$ for an hour, the supernatant liquid of the reaction mixture was removed and concentrated. The residues after decantation and evaporation were dissolved in water and methylene chloride $(25 \mathrm{ml})$, the combined layers were then extracted with methylene chloride $(3 \times 20 \mathrm{ml})$. The organic extract was washed with aqueous sodium hydroxide solution, brine and then dried over anhydrous sodium sulphate. The solvent was distilled off under reduced pressure. The pure product was separated by silica gel column chromatography.

\section{General method for the synthesis of derivatives 25-28 (Scheme 3 )}

The condensation of 1,4-naphthoquinone $\mathbf{7}$ and 3-mercapto propionic acid $\mathbf{1 4}$ in 1:1 ratio in absolute ethanol at room temperature for 3-4 h gave the acid derivative 25 . The isolated acid product 25 was dissolved in dry dichloromethane and equimolar quantity of thionyl chloride was slowly added to it. The mixture was refluxed for $2-3 \mathrm{~h}$ and the acid chloride formation was confirmed by TLC. Then equimolar ratio of primary amines in dry dichloromethane was added to the acid chloride in solution and stirred for 8-9 hours at room temperature. After completion of 
the reaction organic layer was washed with sodium bicarbonate solution, evaporated and the amides formed were purified by column chromatography (60-70\% yields).

2-(3-Acetyl-3H-imidazol-4-yl)naphthalene-1,4-dione (15). Obtained by reaction of naphthalene-1,4-dione $7(0.158 \mathrm{~g}, 1 \mathrm{mmol})$ and 1-acetyl imidazole $(0.110 \mathrm{~g}, 1 \mathrm{mmol})$ to give 15 as dark yellow liquid, yield $0.111 \mathrm{~g}, 42 \% ;{ }^{1} \mathrm{H} \mathrm{NMR}\left(\mathrm{CDCl}_{3}\right): \delta 8.36(\mathrm{~s}, 1 \mathrm{H}), 8.20(\mathrm{~s}, 1 \mathrm{H}), 8.10(\mathrm{~s}$, $1 \mathrm{H}), 7.98-7.74(\mathrm{~m}, 4 \mathrm{H}), 2.24(\mathrm{~s}, 3 \mathrm{H}) ;{ }^{13} \mathrm{C} \mathrm{NMR}\left(\mathrm{CDCl}_{3}\right): \delta 202.2,187.5(2 \mathrm{C}), 155.6,138.0$ (2C), 137.1, 135.3, 134.0 (2C), 130.0 (2C), 124.0 (2C), 18.5; LRMS (ES $\left.{ }^{+}\right): m / z 267.3$ [(M+H) ${ }^{+}$, $100 \%$ ]; Anal. Calc for $\mathrm{C}_{15} \mathrm{H}_{10} \mathrm{~N}_{2} \mathrm{O}_{3}$ (266.25): C, 67.67; H, 3.79; N, 10.52; found: C, 67.78; H, $3.98 ; \mathrm{N}, 10.58$.

2-(5-Acetyl-3-methyl-thiophen-2-yl)naphthalene-1,4-dione (16). Obtained by reaction of naphthalene-1,4-dione $7(0.158 \mathrm{~g}, 1 \mathrm{mmol})$ and 2-acetyl-4-methyl thiophene $(0.140 \mathrm{~g}, 1 \mathrm{mmol})$ as a viscous liquid, yield $0.085 \mathrm{~g}, 29 \% ;{ }^{1} \mathrm{H} \mathrm{NMR}\left(\mathrm{CDCl}_{3}\right): \delta 8.20(\mathrm{~s}, 1 \mathrm{H}), 8.00-7.96(\mathrm{~m}, 4 \mathrm{H})$, $7.64(\mathrm{~s}, 1 \mathrm{H}), 2.58(\mathrm{~s}, 3 \mathrm{H}), 2.20(\mathrm{~s}, 3 \mathrm{H}) ;{ }^{13} \mathrm{C} \mathrm{NMR}\left(\mathrm{CDCl}_{3}\right): \delta 202.4,192.8,187.4(2 \mathrm{C}), 153.5$,

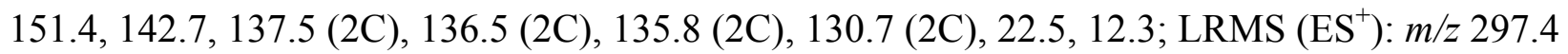
$\left[(\mathrm{M}+\mathrm{H})^{+}, 100 \%\right]$; Anal. Calc for $\mathrm{C}_{17} \mathrm{H}_{12} \mathrm{O}_{3} \mathrm{~S}$ (296.34): C, $68.90 \mathrm{H}, 4.08$; S, 10.82; found: C, 68.92; H, 4.18; S, 10.86 .

5-(1,4-Dioxo-1,4-dihydro-naphthalen-2-yl)-1 $H$-pyrrole-2-carboxylic acid methyl ester (17). Obtained by reaction of naphthalene-1,4-dione $7(0.158 \mathrm{~g}, 1 \mathrm{mmol})$ and methyl-2-pyrrole carboxylate $(0.110 \mathrm{~g}, 1 \mathrm{mmol})$ as a liquid, yield $0.090 \mathrm{~g}, 32 \% ;{ }^{1} \mathrm{H} \mathrm{NMR}\left(\mathrm{CDCl}_{3}\right): \delta 8.10(\mathrm{~s}, 1 \mathrm{H})$, $7.96-7.80(\mathrm{~m}, 4 \mathrm{H}), 6.74(\mathrm{~d}, 1 \mathrm{H}, \mathrm{J}=7.40 \mathrm{~Hz}), 6.22(\mathrm{~d}, 1 \mathrm{H}, \mathrm{J}=7.42 \mathrm{~Hz}), 4.00(\mathrm{~s}, 3 \mathrm{H}) ;{ }^{13} \mathrm{C}$ NMR $\left(\mathrm{CDCl}_{3}\right): \delta 187.4(2 \mathrm{C}), 160.2,153.5,137.4(2 \mathrm{C}), 136.8,134.0$ (2C), $130.6(2 \mathrm{C}), 123.5(2 \mathrm{C})$, 116.0, 112.4, 53.2; LRMS $\left(\mathrm{ES}^{+}\right): \mathrm{m} / z 282.3\left[(\mathrm{M}+\mathrm{H})^{+}, 100 \%\right]$; Anal. Calc for $\mathrm{C}_{16} \mathrm{H}_{11} \mathrm{NO}_{4}$ (281.26): C, 68.32; H, 3.94; N, 4.98; found: C, 68.40; H, 3.99; N, 5.01.

2-(1,4-Dioxo-1,4-dihydro-naphthalen-2-yl)-pyrrole-1-carboxylic acid methyl ester (18). Obtained by reaction of naphthalene-1,4-dione $7(0.158 \mathrm{~g}, 1 \mathrm{mmol})$ and methyl-1-pyrrole carboxylate $(0.125 \mathrm{~g}, 1 \mathrm{mmol})$ as a liquid, yield $0.118 \mathrm{~g}, 42 \% ;{ }^{1} \mathrm{H} \mathrm{NMR}\left(\mathrm{CDCl}_{3}\right): \delta 8.14(\mathrm{~s}, 1 \mathrm{H})$, $7.98-7.84(\mathrm{~m}, 4 \mathrm{H}), 6.68(\mathrm{~d}, 1 \mathrm{H}, \mathrm{J}=7.45 \mathrm{~Hz}), 6.20(\mathrm{~m}, 2 \mathrm{H}), 3.72(\mathrm{~s}, 3 \mathrm{H}) ;{ }^{13} \mathrm{C} \mathrm{NMR}\left(\mathrm{CDCl}_{3}\right): \delta$ 187.3 (2C), 160.4, 153.5, 137.4 (2C), 136.8, 134.8 (2C), 130.6 (2C), 119.2 (2C), 111.2 (2C), 18.0; LRMS $\left(\mathrm{ES}^{+}\right): m / z 282.4\left[(\mathrm{M}+\mathrm{H})^{+}, 100 \%\right]$; Anal. Calc for $\mathrm{C}_{16} \mathrm{H}_{11} \mathrm{NO}_{4}$ (281.26): C, 68.32; H, 3.94; N, 4.98; found: C, 68.38; H, 4.04; N, 5.00.

2-(1-Methyl-6-oxo-1,6-dihydro-pyridin-3-yl)naphthalene-1,4-dione (19). Obtained by reaction of naphthalene-1,4-dione $7(0.158 \mathrm{~g}, 1 \mathrm{mmol})$ and 1-methyl-2-pyridone $(0.110 \mathrm{~g}$, $1 \mathrm{mmol})$ as a viscous liquid, yield $0.092 \mathrm{~g}, 35 \% ;{ }^{1} \mathrm{H} \mathrm{NMR}\left(\mathrm{CDCl}_{3}\right): \delta 8.12-7.98(\mathrm{~m}, 4 \mathrm{H}), 7.82$ $(\mathrm{s}, 1 \mathrm{H}), 7.42(\mathrm{~s}, 1 \mathrm{H}), 7.02(\mathrm{~s}, 1 \mathrm{H}), 6.54(\mathrm{~s}, 1 \mathrm{H}), 2.82(\mathrm{~s}, 3 \mathrm{H}) ;{ }^{13} \mathrm{C} \mathrm{NMR}\left(\mathrm{CDCl}_{3}\right): \delta 188.0(2 \mathrm{C})$, 161.8, 155.2, 144.7, 138.2 (2C), 135.0 (2C), 134.4, 132.0 (2C), 126.8, 122.8, 115.4, 35.0; LRMS $\left(\mathrm{ES}^{+}\right): m / z 266.3\left[(\mathrm{M}+\mathrm{H})^{+}, 100 \%\right]$; Anal. Calc for $\mathrm{C}_{16} \mathrm{H}_{11} \mathrm{NO}_{3}$ (265.26): C, 72.45; H, 4.18; N, 5.28; found: C, 72.55; H, 4.23; N, 5.33 .

2-(4-Methyl-pyrimidin-5-yl)naphthalene-1,4-dione (20). Obtained by reaction of naphthalene1,4-dione 7 ( $0.158 \mathrm{~g}, 1 \mathrm{mmol})$ and 4-methyl pyrimidine $(0.095 \mathrm{~g}, 1 \mathrm{mmol})$ as a viscous oil, yield $0.065 \mathrm{~g}, 26 \% ;{ }^{1} \mathrm{H}$ NMR $\left(\mathrm{CDCl}_{3}\right): \delta 8.98(\mathrm{~s}, 1 \mathrm{H}), 8.76(\mathrm{~s}, 1 \mathrm{H}), 8.10(\mathrm{~s}, 1 \mathrm{H}), 7.96-7.80(\mathrm{~m}, 4 \mathrm{H})$, 
$2.42(\mathrm{~s}, 3 \mathrm{H}) ;{ }^{13} \mathrm{C} \mathrm{NMR}\left(\mathrm{CDCl}_{3}\right): \delta 187.8(2 \mathrm{C}), 164.2,158.2,154.6,152.7,138.2(2 \mathrm{C}), 137.2$, 135.2 (2C), 130.4 (2C), 127.5, 16.6; LRMS (ES $\left.{ }^{+}\right): m / z 251.3\left[(\mathrm{M}+\mathrm{H})^{+}, 100 \%\right]$; Anal. Calc for $\mathrm{C}_{15} \mathrm{H}_{10} \mathrm{~N}_{2} \mathrm{O}_{2}$ (250.25): C, 71.99; H, 4.03; N, 11.19; found: C, 72.08; H, 4.13; N, 11.25.

3-(1,4-Dioxo-1,4-dihydro-naphthalen-2-yl)-acrylic acid ethyl ester (21). Obtained from the oxidation of 3-naphthalen-2-yl-acrylic acid ethyl ester $9(0.226 \mathrm{~g}, 1 \mathrm{mmol})$ with periodic acid $(0.957 \mathrm{~g}, 4.2 \mathrm{mmol})$ and chromium trioxide $(0.015 \mathrm{~g}, 0.1 \mathrm{mmol})$ as $0.133 \mathrm{~g}$ viscous liquid in $52 \%$ yield. IR $\left(\mathrm{KBr}, \mathrm{cm}^{-1}\right): 3055,1730,1715,1660,1645,1435,1313,1273,1192 ;{ }^{1} \mathrm{H}$ NMR $\left(\mathrm{CDCl}_{3}\right): \delta 7.98-7.94(\mathrm{~m}, 2 \mathrm{H}), 7.85-7.84(\mathrm{~m}, 3 \mathrm{H}), 7.20(\mathrm{~d}, 1 \mathrm{H}, \mathrm{J}=8.4 \mathrm{~Hz}), 6.28(\mathrm{~s}, 1 \mathrm{H}), 4.20$ $(\mathrm{m}, 2 \mathrm{H}), 1.35(\mathrm{~s}, 3 \mathrm{H}) ;{ }^{13} \mathrm{C} \mathrm{NMR}\left(\mathrm{CDCl}_{3}\right): \delta 188.0(2 \mathrm{C}), 166.4,154.4,145.7,138.0(2 \mathrm{C}), 135.0$ (2C), 134.6, 130.4 (2C), 122.0, 60.8, 15.6; LRMS (ES $\left.{ }^{+}\right): m / z 257.3\left[(\mathrm{M}+\mathrm{H})^{+}, 100 \%\right]$; Anal. Calc for $\mathrm{C}_{15} \mathrm{H}_{12} \mathrm{O}_{4}$ (256.25): C, 70.31; H, 4.72; found: C, 70.43; H, 4.79 .

2-(1,4-Dioxo-1,4-dihydro-naphthalen-2-yl)-3-methyl-but-2-enoic acid ethyl ester (22). Obtained from the oxidation of 3-methyl-2-naphthalen-2-yl-but-2-enoic acid ethyl ester 11 $(0.254 \mathrm{~g}, 1 \mathrm{mmol})$ with periodic acid $(0.957 \mathrm{~g}, 4.2 \mathrm{mmol})$ and chromium trioxide $(0.015 \mathrm{~g}$, $0.1 \mathrm{mmol})$ as $0.170 \mathrm{~g}$ viscous liquid in $60 \%$ yield. IR $\left(\mathrm{KBr}, \mathrm{cm}^{-1}\right): 3055,2925,1736,1728,1665$, 1645, 1590, 1546, 1432; ${ }^{1} \mathrm{H}$ NMR $\left(\mathrm{CDCl}_{3}\right): \delta 8.02$ - $7.98(\mathrm{~m}, 2 \mathrm{H}), 7.84-7.76(\mathrm{~m}, 3 \mathrm{H}), 4.20(\mathrm{~m}$, $2 \mathrm{H}), 1.86(\mathrm{~s}, 6 \mathrm{H}), 1.36(\mathrm{~s}, 3 \mathrm{H}) ;{ }^{13} \mathrm{C} \mathrm{NMR}\left(\mathrm{CDCl}_{3}\right): \delta 187.8(2 \mathrm{C}), 172.2,155.2,142.8,138.8$

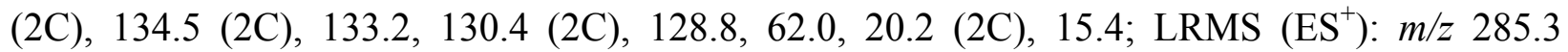
$\left[(\mathrm{M}+\mathrm{H})^{+}, 100 \%\right]$; Anal. Calc for $\mathrm{C}_{17} \mathrm{H}_{16} \mathrm{O}_{4}$ (284.31): C, 71.82; H, 5.67; found: C, 71.98; H, 5.78.

Propionic acid 2-(1,4-dioxo-1,4-dihydro-naphthalen-2-yl)-1-methyl vinyl ester (23). Obtained from the oxidation of propionic acid-1-methyl-2-naphthalen-2-yl-vinyl ester 13 (0. $240 \mathrm{~g}, 1 \mathrm{mmol})$ with periodic acid $(0.957 \mathrm{~g}, 4.2 \mathrm{mmol})$ and chromium trioxide $(0.015 \mathrm{~g}$, $0.1 \mathrm{mmol})$ as $0.130 \mathrm{~g}$ liquid in $48 \%$ yield. IR $\left(\mathrm{KBr}, \mathrm{cm}^{-1}\right): 3054,2852,1754,1715,1662,1631$, 1598, 1455, 1368, 1217; ${ }^{1} \mathrm{H}$ NMR $\left(\mathrm{CDCl}_{3}\right): \delta 8.00-7.98(\mathrm{~m}, 4 \mathrm{H}), 7.82(\mathrm{~s}, 1 \mathrm{H}), 6.00(\mathrm{~s}, 1 \mathrm{H})$, $2.34(\mathrm{~m}, 2 \mathrm{H}), 2.04(\mathrm{~s}, 3 \mathrm{H}), 1.24(\mathrm{~s}, 3 \mathrm{H}) ;{ }^{13} \mathrm{C} \mathrm{NMR}\left(\mathrm{CDCl}_{3}\right): \delta 188.2(2 \mathrm{C}), 168.4,155.2,145.4$, 138.2 (2C), 134.5 (2C), 133.3, 130.4 (2C), 104.2, 28.8, 18.1, 12.2; LRMS (ES $\left.{ }^{+}\right): m / z 271.4$ $\left[(\mathrm{M}+\mathrm{H})^{+}, 100 \%\right]$; Anal. Calc for $\mathrm{C}_{16} \mathrm{H}_{14} \mathrm{O}_{4}$ (270.28): C, 71.10; H, 5.22; found: C, 71.23; H, 5.28.

3-(1,4-Dioxo-1,4-dihydro-naphthalen-2-yl)-but-2-enoic acid methyl ester (24). Obtained from the oxidation of 3-naphthalen-2-yl-but-2-enoic acid methyl ester $\mathbf{1 0}(0.226 \mathrm{~g}, 1 \mathrm{mmol})$ with periodic acid $(0.957 \mathrm{~g}, 4.2 \mathrm{mmol})$ and chromium trioxide $(0.015 \mathrm{~g}, 0.1 \mathrm{mmol})$ as $0.133 \mathrm{~g}$ viscous liquid in $52 \%$ yield. IR $\left(\mathrm{KBr}, \mathrm{cm}^{-1}\right)$ : 3057, 2854, 1730, 1714, 1662, 1629, 1504; ${ }^{1} \mathrm{H}$ NMR $\left(\mathrm{CDCl}_{3}\right): \delta 7.98-7.94(\mathrm{~m}, 2 \mathrm{H}), 7.84-7.80(\mathrm{~m}, 3 \mathrm{H}), 6.28(\mathrm{~s}, 1 \mathrm{H}), 3.85(\mathrm{~s}, 3 \mathrm{H}), 1.86(\mathrm{~s}, 3 \mathrm{H}) ;{ }^{13} \mathrm{C}$ NMR $\left(\mathrm{CDCl}_{3}\right): \delta 187.2$ (2C), 165.4, 155.4, 150.4, 138.1 (2C), 134.5 (2C), 133.8, 130.5 (2C), 116.4, 51.2, 15.5; LRMS (ES $\left.{ }^{+}\right): m / z 257.3\left[(\mathrm{M}+\mathrm{H})^{+}, 100 \%\right]$; Anal. Calc for $\mathrm{C}_{15} \mathrm{H}_{12} \mathrm{O}_{4}(256.25)$ : C, 70.31; H, 4.72; found: C, 70.44; H, 4.84.

3-(1,4-Dioxo-1,4-dihydro-naphthalen-2-ylsulfanyl)-propionic acid (25). Obtained by condensation of naphthalene-1,4-dione $7(0.158 \mathrm{~g}, 1 \mathrm{mmol})$ and 3-mercapto propionic acid 14 $(0.107 \mathrm{~g}, 1 \mathrm{mmol})$ as $0.157 \mathrm{~g}$ brown solid in $60 \%$ yield (recrystallized from methanol); mp. 105$106{ }^{0} \mathrm{C}$;. IR (KBr, cm $\left.{ }^{-1}\right): 3400,1688,669 ;{ }^{1} \mathrm{H} \mathrm{NMR}\left(\mathrm{CDCl}_{3}\right): \delta 8.13(\mathrm{~m}, 2 \mathrm{H}, 5,8-\mathrm{ArH}), 7.79(\mathrm{~m}$, $2 \mathrm{H}, 6,7-\mathrm{ArH}), 6.67(\mathrm{~s}, 1 \mathrm{H}, 3-\mathrm{Naph}-\mathrm{H}), 3.14$ (t, 2H, $\left.\mathrm{SCH}_{2}, J=7.12 \mathrm{~Hz}\right), 2.84\left(\mathrm{t}, 2 \mathrm{H}, \mathrm{CH}_{2} \mathrm{C}=\mathrm{O}, \mathrm{J}\right.$ 
$=7.20 \mathrm{~Hz}) ;{ }^{13} \mathrm{C} \mathrm{NMR}\left(\mathrm{CDCl}_{3}\right): \delta 188.4(2 \mathrm{C}), 178.4,161.1,138.3(2 \mathrm{C}), 134.8(2 \mathrm{C}), 130.8(2 \mathrm{C})$, 125.2, 38.8, 26.4; LRMS $\left(\mathrm{ES}^{+}\right): m / z 263.3\left[(\mathrm{M}+\mathrm{H})^{+}, 100 \%\right.$ ]; Anal. Calc for $\mathrm{C}_{13} \mathrm{H}_{10} \mathrm{O}_{4} \mathrm{~S}(262.28)$ : C, 59.53; H, 3.84; S, 12.23; found: C, 59.62; H, 3.98; S, 12.31 .

3-(1,4-Dioxo-1,4-dihydro-naphthalen-2-ylsulfanyl)-propionic acid ethyl ester (26). Obtained from the esterification of $\mathbf{2 5}(0.262 \mathrm{~g}, 1 \mathrm{mmol})$ with ethanol as $0.190 \mathrm{~g}$ oil in $66 \%$ yield. IR ( $\mathrm{KBr}$, $\left.\mathrm{cm}^{-1}\right): 3400,1728,1675,670 ;{ }^{1} \mathrm{H}$ NMR $\left(\mathrm{CDCl}_{3}\right): \delta 8.08(\mathrm{~m}, 2 \mathrm{H}, 5,8-\mathrm{ArH}), 7.80(\mathrm{~m}, 2 \mathrm{H}, 6,7-$ $\mathrm{ArH}), 6.87(\mathrm{~s}, 1 \mathrm{H}, 3-\mathrm{Naph}-\mathrm{H}), 4.18(\mathrm{~m}, 2 \mathrm{H}), 3.22\left(\mathrm{t}, 2 \mathrm{H}, \mathrm{SCH}_{2}, J=7.12 \mathrm{~Hz}\right), 2.66$ (t, 2H, $\left.\mathrm{CH}_{2} \mathrm{C}=\mathrm{O}, \mathrm{J}=7.20 \mathrm{~Hz}\right), 1.34(\mathrm{t}, 3 \mathrm{H}, \mathrm{J}=7.00 \mathrm{~Hz}) ;{ }^{13} \mathrm{C} \mathrm{NMR}\left(\mathrm{CDCl}_{3}\right): \delta 188.4(2 \mathrm{C}), 172.4,161.2$, 138.4 (2C), 134.8 (2C), 130.7 (2C), 124.2, 60.4, 35.8, 25.6, 15.0; LRMS (ES $\left.{ }^{\dagger}\right): m / z 290.9\left[(\mathrm{M})^{+}\right.$, 100\%]; Anal. Calc for $\mathrm{C}_{15} \mathrm{H}_{14} \mathrm{O}_{4} \mathrm{~S}$ (290.34): C, 62.05; H, 4.86; S, 11.04; found: C, 62.12; H, 4.97; S, 11.10 .

$\mathrm{N}$-Benzyl-3-(1,4-dioxo-1,4-dihydro-naphthalen-2-ylsulfanyl)-propionamide (27). Obtained from the reaction of $25(0.262 \mathrm{~g}, 1 \mathrm{mmol})$ with benzyl amine $(0.107 \mathrm{~g}, 1 \mathrm{mmol})$ as $0.245 \mathrm{~g}$ brown solid in $70 \%$ yield (recrystallized from methanol); mp. $165-167^{0} \mathrm{C}$; IR $\left(\mathrm{KBr}, \mathrm{cm}^{-1}\right): 3401,3000$, 1640, 669; ${ }^{1} \mathrm{H}$ NMR $\left(\mathrm{CDCl}_{3}\right): \delta 8.09$ (m, 2H, 5,8-ArH), 7.77 (m, 2H, 6,7-ArH), $7.32-7.28(\mathrm{~m}$, 5H-Ar), 6.88 (s, 1H, 3-Naph-H), 3.49 (s, 2H, $\left.\mathrm{CH}_{2}-\mathrm{Ph}\right), 3.21$ (t, 2H, $\mathrm{SCH}_{2}, J=7.10 \mathrm{~Hz}$ ), 2.65 (t, $\left.2 \mathrm{H}, \mathrm{CH}_{2} \mathrm{C}=\mathrm{O}, \mathrm{J}=7.0 \mathrm{~Hz}\right) ;{ }^{13} \mathrm{C} \mathrm{NMR}\left(\mathrm{CDCl}_{3}\right): \delta 188.2(2 \mathrm{C}), 176.4,160.7,138.2(2 \mathrm{C}), 134.4$ (2C), 130.8 (2C), 145.5, 129.0 (2C), 127.5 (2C), 124.2, 112.8, 50.0, 37.6, 25.6; LRMS (ES $\left.{ }^{+}\right): \mathrm{m} / \mathrm{z}$ $352.5\left[(\mathrm{M}+\mathrm{H})^{+}, 100 \%\right]$; Anal. Calc for $\mathrm{C}_{20} \mathrm{H}_{17} \mathrm{NO}_{3} \mathrm{~S}$ (351.42): C, 68.36; $\mathrm{H}, 4.88$; N, 3.99; found: C, 68.43; H, 5.00; N, 4.02 .

2-(3-(4-Methyl-piperazin-1-yl)-3-oxo-propylsulfanyl)naphthalene-1,4-dione (28). Obtained from the reaction of $25(0.262 \mathrm{~g}, 1 \mathrm{mmol})$ with 1-methyl piperazine $(0.100 \mathrm{~g}, 1 \mathrm{mmol})$ as $0.237 \mathrm{~g}$ brown solid in $69 \%$ yield (recrystallized from methanol); mp. $110-112{ }^{0} \mathrm{C}$; IR $\left(\mathrm{KBr}, \mathrm{cm}^{-1}\right)$ : 3404 , 3010, 1642, 670; ${ }^{1} \mathrm{H}$ NMR $\left(\mathrm{CDCl}_{3}\right): \delta 8.10(\mathrm{~m}, 2 \mathrm{H}, 5,8-\mathrm{ArH}), 7.76(\mathrm{~m}, 2 \mathrm{H}, 6,7-\mathrm{ArH}), 6.64(\mathrm{~s}$, 1H, 3-Naph-H), $3.90(\mathrm{~s}, 3 \mathrm{H}), 3.48(\mathrm{~m}, 4 \mathrm{H}), 3.13\left(\mathrm{t}, 2 \mathrm{H}, \mathrm{SCH}_{2}, J=7.10 \mathrm{~Hz}\right), 2.84(\mathrm{t}, 2 \mathrm{H}$, $\left.\mathrm{CH}_{2} \mathrm{C}=\mathrm{O}, \mathrm{J}=7.20 \mathrm{~Hz}\right), 2.68(\mathrm{~m}, 4 \mathrm{H}) ;{ }^{13} \mathrm{C} \mathrm{NMR}\left(\mathrm{CDCl}_{3}\right): \delta 188.0(2 \mathrm{C}), 174.0,160.6,138.4(2 \mathrm{C})$, 134.5 (2C), 131.0 (2C), 124.2, 58.2 (2C), 50.4 (2C), 38.2, 35.2, 27.2; LRMS (ES $\left.{ }^{+}\right): m / z 345.5$ $\left[(\mathrm{M}+\mathrm{H})^{+}, 100 \%\right]$; Anal. Calc for $\mathrm{C}_{18} \mathrm{H}_{20} \mathrm{~N}_{2} \mathrm{O}_{3} \mathrm{~S}$ (344.43): C, 62.77; H, 5.85; N, 8.13; S, 9.31; found: C, 62.81; H, 5.95; N, 8.20; S, 9.36.

2-(1-Acetyl-1H-indol-3-yl)naphthalene-1,4-dione (29). Obtained by reaction of naphthalene1,4-dione $7(0.158 \mathrm{~g}, 1 \mathrm{mmol})$ and 1-acetyl indole $(0.162 \mathrm{~g}, 1 \mathrm{mmol})$ as a liquid, yield $0.095 \mathrm{~g}$, 30\%; ${ }^{1} \mathrm{H}$ NMR $\left(\mathrm{CDCl}_{3}\right): \delta 8.90(\mathrm{~s}, 1 \mathrm{H}), 8.20(\mathrm{~s}, 1 \mathrm{H}), 8.08(\mathrm{~m}, 2 \mathrm{H}), 7.96(\mathrm{~m}, 2 \mathrm{H}), 6.98(\mathrm{~m}, 4 \mathrm{H})$, $2.24(\mathrm{~s}, 3 \mathrm{H}) ;{ }^{13} \mathrm{C} \mathrm{NMR}\left(\mathrm{CDCl}_{3}\right): \delta 198.2,188.2(2 \mathrm{C}), 154.2,138.0(2 \mathrm{C}), 136.0(2 \mathrm{C}), 135.2(2 \mathrm{C})$,

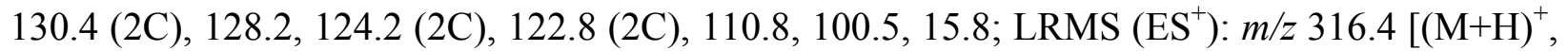
100\%]; Anal. Calc for $\mathrm{C}_{20} \mathrm{H}_{13} \mathrm{NO}_{3}$ (315.32): C, 76.18; H, 4.16; N, 4.44; found: C, 76.26; H, 4.23; $\mathrm{N}, 4.61$.

Thioacetic acid-5-(1,4-dioxo-1,4-dihydro-naphthalen-2-yl)furan-2-ylmethyl ester (30). Obtained by reaction of naphthalene-1,4-dione $7(0.158 \mathrm{~g}, 1 \mathrm{mmol})$ and S-furfuryl thioacetate $(0.158 \mathrm{~g}, 1 \mathrm{mmol})$ as an oil, yield $0.100 \mathrm{~g}, 32 \% ;{ }^{1} \mathrm{H} \mathrm{NMR}\left(\mathrm{CDCl}_{3}\right): \delta 8.20(\mathrm{~s}, 1 \mathrm{H}), 8.08(\mathrm{~m}, 2 \mathrm{H})$, $7.96(\mathrm{~m}, 2 \mathrm{H}), 7.59(\mathrm{~s}, 1 \mathrm{H}), 7.37(\mathrm{~d}, 1 \mathrm{H}, \mathrm{J}=4.22 \mathrm{~Hz}), 4.16(\mathrm{~s}, 2 \mathrm{H}), 2.38(\mathrm{~s}, 3 \mathrm{H}) ;{ }^{13} \mathrm{C} \mathrm{NMR}$ 
$\left(\mathrm{CDCl}_{3}\right): \delta 198.8,187.6(2 \mathrm{C}), 155.2,154.0(2 \mathrm{C}), 138.2$ (2C), 136.0, $134.1(2 \mathrm{C}), 130.4(2 \mathrm{C})$, 115.5, 110.2, 32.8, 28.2; LRMS $\left(\mathrm{ES}^{+}\right): m / z 313.4\left[(\mathrm{M}+\mathrm{H})^{+}, 100 \%\right]$; Anal. Calc for $\mathrm{C}_{17} \mathrm{H}_{12} \mathrm{O}_{4} \mathrm{~S}$ (312.34): C, 65.37; H, 3.87; S, 10.27; found: C, 65.39; H, 3.99; S, 10.25.

2-(5-Acetyl-furan-2-yl) naphthalene-1,4-dione (31). Obtained by reaction of naphthalene-1,4dione $7(0.158 \mathrm{~g}, 1 \mathrm{mmol})$ and 2-acetyl furan $(0.110 \mathrm{~g}, 1 \mathrm{mmol})$ as a viscous liquid, yield $0.069 \mathrm{~g}, 26 \% ;{ }^{1} \mathrm{H}$ NMR $\left(\mathrm{CDCl}_{3}\right): \delta 8.18(\mathrm{~s}, 1 \mathrm{H}), 8.08(\mathrm{~m}, 2 \mathrm{H}), 7.97(\mathrm{~m}, 2 \mathrm{H}), 7.61(\mathrm{~d}, 1 \mathrm{H}, \mathrm{J}=$ $4.18 \mathrm{~Hz}), 7.29(\mathrm{~d}, 1 \mathrm{H}, \mathrm{J}=4.22 \mathrm{~Hz}), 2.60(\mathrm{~s}, 3 \mathrm{H}) ;{ }^{13} \mathrm{C} \mathrm{NMR}\left(\mathrm{CDCl}_{3}\right): \delta 188.0(2 \mathrm{C}), 184.8,160.2$, 155.0, 152.5, 138.4 (2C), 137.0, 134.8 (2C), 131.2 (2C), 126.6, 115.8, 21.2; LRMS (ES $\left.{ }^{+}\right): m / z$ $267.3\left[(\mathrm{M}+\mathrm{H})^{+}, 100 \%\right]$; Anal. Calc for $\mathrm{C}_{16} \mathrm{H}_{10} \mathrm{O}_{4}$ (266.25): C, 72.18; H, 3.79; found: C, 72.11; H, 3.97 .

2-(1,4-Dioxo-1,4-dihydro-naphthalen-2-yl)-3-methyl-but-2-enoic acid methyl ester (32). Obtained from the oxidation of 3-methyl-2-naphthalen-2-yl-but-2-enoic acid methyl ester 12 (0. $240 \mathrm{~g}, 1 \mathrm{mmol})$ with periodic acid $(0.957 \mathrm{~g}, 4.2 \mathrm{mmol})$ and chromium trioxide $(0.015 \mathrm{~g}$, $0.1 \mathrm{mmol})$ as $0.149 \mathrm{~g}$ viscous liquid in $55 \%$ yield. IR $\left(\mathrm{KBr}, \mathrm{cm}^{-1}\right): 3055,2925,1736,1728,1645$, 1591, 1548, 1436; ${ }^{1} \mathrm{H}$ NMR $\left(\mathrm{CDCl}_{3}\right): \delta 8.02$ - $7.98(\mathrm{~m}, 2 \mathrm{H}), 7.84$ - $7.76(\mathrm{~m}, 3 \mathrm{H}), 3.67(\mathrm{~s}, 3 \mathrm{H})$, $1.88(\mathrm{~s}, 6 \mathrm{H}) ;{ }^{13} \mathrm{C} \mathrm{NMR}\left(\mathrm{CDCl}_{3}\right): \delta 188.2(2 \mathrm{C}), 155.0,142.2,138.2(2 \mathrm{C}), 135.0(2 \mathrm{C}), 132.7$, 130.6 (2C), 128.0, 60.0, 20.0; LRMS (ES $\left.{ }^{+}\right): m / z 271.3\left[(\mathrm{M}+\mathrm{H})^{+}, 100 \%\right.$ ]; Anal. Calc for $\mathrm{C}_{14} \mathrm{H}_{14} \mathrm{O}_{4}$ (270.28): C, 71.10; H, 5.22; found: C, 71.28; H, 5.30.

5-(1,4-Dioxo-1,4-dihydro-naphthalen-2-yl)-1-methyl-1 $H$-pyrrol-2-yl-acetic acid methyl ester (33). Obtained by reaction of naphthalene-1,4-dione 7 (0.158 g, $1 \mathrm{mmol})$ and methyl-1methyl-2-pyrrole acetate $(0.153 \mathrm{~g}, 1 \mathrm{mmol})$ as a liquid, yield $0.087 \mathrm{~g}, 28 \% ;{ }^{1} \mathrm{H} \mathrm{NMR}\left(\mathrm{CDCl}_{3}\right): \delta$ $8.22(\mathrm{~s}, 1 \mathrm{H}), 8.08(\mathrm{~m}, 2 \mathrm{H}), 7.98(\mathrm{~m}, 2 \mathrm{H}), 7.08(\mathrm{~d}, 1 \mathrm{H}, \mathrm{J}=7.45 \mathrm{~Hz}), 6.56(\mathrm{~d}, 1 \mathrm{H}, \mathrm{J}=7.40 \mathrm{~Hz})$, $4.24(\mathrm{~s}, 2 \mathrm{H}), 3.66(\mathrm{~s}, 3 \mathrm{H}), 2.46(\mathrm{~s}, 3 \mathrm{H}) ;{ }^{13} \mathrm{C} \mathrm{NMR}\left(\mathrm{CDCl}_{3}\right): \delta 187.8(2 \mathrm{C}), 172.0,154.3,138.5$ (2C), 136.9, 134.4 (2C), 133.4, 130.1 (2C), 133.2, 110.1 (2C), 51.5, 28.4, 25.6; LRMS (ES $\left.{ }^{+}\right): m / z$ $310.4\left[(\mathrm{M}+\mathrm{H})^{+}, 100 \%\right]$; Anal. Calc for $\mathrm{C}_{18} \mathrm{H}_{15} \mathrm{NO}_{4}$ (309.32): C, 69.89; H, 4.89; N, 4.53; found: C, 69.94; H, 4.99; N, 4.49.

2-(4-Acetyl-1-methyl-1H-pyrrol-2-yl) naphthalene-1,4-dione (34). Obtained by reaction of naphthalene-1,4-dione $7(0.158 \mathrm{~g}, 1 \mathrm{mmol})$ and 3-acetyl-1-methyl pyrrole $(0.123 \mathrm{~g}, 1 \mathrm{mmol})$ as a viscous liquid, yield $0.086 \mathrm{~g}, 31 \% ;{ }^{1} \mathrm{H} \mathrm{NMR}\left(\mathrm{CDCl}_{3}\right): \delta 8.20(\mathrm{~s}, 1 \mathrm{H}), 8.08(\mathrm{~m}, 2 \mathrm{H}), 7.98(\mathrm{~m}, 2 \mathrm{H})$, $7.28(\mathrm{~s}, 1 \mathrm{H}), 6.56(\mathrm{~s}, 1 \mathrm{H}), 3.66(\mathrm{~s}, 3 \mathrm{H}), 2.66(\mathrm{~s}, 3 \mathrm{H}) ;{ }^{13} \mathrm{C} \mathrm{NMR}\left(\mathrm{CDCl}_{3}\right): \delta 198.5,188.1(2 \mathrm{C})$, 154.2, 138.1 (2C), 135.1 (2C), 137.0, 131.3 (2C), 124.0 (2C), 106.6 (2C), 35.5, 23.5; LRMS $\left(\mathrm{ES}^{+}\right): \mathrm{m} / \mathrm{z} 280.3\left[(\mathrm{M}+\mathrm{H})^{+}, 100 \%\right.$; Anal. Calc for $\mathrm{C}_{17} \mathrm{H}_{13} \mathrm{NO}_{3}$ (279.29): C, 73.11; H, 4.69; N, 5.02; found: C, 72.99; H, 4.82; N, 4.99 .

2-(6-Methyl-5-methylsulfanyl-pyrazin-2-yl) naphthalene-1,4-dione (35). Obtained by reaction of naphthalene-1,4-dione $7(0.158 \mathrm{~g}, 1 \mathrm{mmol})$ and 2-methyl-3-(methylthio) pyrazine $(0.140 \mathrm{~g}$, $1 \mathrm{mmol})$ as an oil, yield $0.077 \mathrm{~g}, 26 \% ;{ }^{1} \mathrm{H} \mathrm{NMR}\left(\mathrm{CDCl}_{3}\right): \delta 8.46(\mathrm{~s}, 1 \mathrm{H}), 8.22(\mathrm{~s}, 1 \mathrm{H}), 8.08(\mathrm{~m}$, 2H), $7.98(\mathrm{~m}, 2 \mathrm{H}), 2.50(\mathrm{~s}, 3 \mathrm{H}), 2.38(\mathrm{~s}, 3 \mathrm{H}) ;{ }^{13} \mathrm{C} \mathrm{NMR}\left(\mathrm{CDCl}_{3}\right): \delta 188.2(2 \mathrm{C}), 154.3(2 \mathrm{C})$, 150.8, 146.2, 141.8, 138.1 (2C), 137.1, 134.5 (2C), 131.2 (2C), 20.8, 15.2; LRMS (ES $\left.{ }^{+}\right): m / z$ $297.4\left[(\mathrm{M}+\mathrm{H})^{+}, 100 \%\right]$; Anal. Calc for $\mathrm{C}_{16} \mathrm{H}_{12} \mathrm{~N}_{2} \mathrm{O}_{2} \mathrm{~S}$ (296.34): C, 64.85; H, 4.08; N, 9.45; S, 10.82; found: C, 64.97; H, 4.18; N, 9.32; S, 10.86 . 
6-(1,4-Dioxo-1,4-dihydro-naphthalen-2-yl)-pyridine-2-carboxylic acid methyl ester (36). Obtained by reaction of naphthalene-1,4-dione $7(0.158 \mathrm{~g}, 1 \mathrm{mmol})$ and methyl picolinate $(0.137 \mathrm{~g}, 1 \mathrm{mmol})$ as an oil, yield $0.076 \mathrm{~g}, 26 \%$; ${ }^{1} \mathrm{H}$ NMR $\left(\mathrm{CDCl}_{3}\right): \delta 8.72(\mathrm{~s}, 1 \mathrm{H}), 8.22-8.12$ $(\mathrm{m}, 3 \mathrm{H}), 8.08(\mathrm{~m}, 2 \mathrm{H}), 7.97(\mathrm{~m}, 2 \mathrm{H}), 3.78(\mathrm{~s}, 3 \mathrm{H}) ;{ }^{13} \mathrm{C} \mathrm{NMR}\left(\mathrm{CDCl}_{3}\right): \delta 188.2(2 \mathrm{C}), 168.2$, 158.2, 156.1, 148.8, 139.1, 138.1 (2C), 137.2, 135.5 (2C), 131.5 (2C), 125.6, 123.8, 51.2; LRMS $\left(\mathrm{ES}^{+}\right): m / z 294.4\left[(\mathrm{M}+\mathrm{H})^{+}, 100 \%\right]$; Anal. Calc for $\mathrm{C}_{17} \mathrm{H}_{11} \mathrm{NO}_{4}$ (293.27): C, 69.62; H, 3.78; N, 4.78; found: C, 69.22; H, 4.02; N, 4.69.

2-(6-Methyl-pyrazin-2-yl) naphthalene-1,4-dione (37). Obtained by reaction of naphthalene1,4-dione $7(0.158 \mathrm{~g}, 1 \mathrm{mmol})$ and 2-methyl pyrazine $(0.095 \mathrm{~g}, 1 \mathrm{mmol})$ as a liquid, yield $0.075 \mathrm{~g}, 30 \%$; ${ }^{1} \mathrm{H}$ NMR $\left(\mathrm{CDCl}_{3}\right): \delta 8.70(\mathrm{~s}, 1 \mathrm{H}), 8.50(\mathrm{~s}, 1 \mathrm{H}), 8.18(\mathrm{~s}, 1 \mathrm{H}), 8.08(\mathrm{~m}, 2 \mathrm{H}), 7.97$ $(\mathrm{m}, 2 \mathrm{H}), 2.40(\mathrm{~s}, 3 \mathrm{H}) ;{ }^{13} \mathrm{C} \mathrm{NMR}\left(\mathrm{CDCl}_{3}\right): \delta 188.2(2 \mathrm{C}), 154.2(2 \mathrm{C}), 150.6,145.0,140.2,137.6$, 138.1 (2C), 134.8 (2C), 131.2 (2C), 22.5; LRMS (ES $\left.{ }^{+}\right): m / z 251.3$ [(M+H) ${ }^{+}, 100 \%$; Anal. Calc for $\mathrm{C}_{15} \mathrm{H}_{10} \mathrm{~N}_{2} \mathrm{O}_{2}$ (250.25): C, 71.99; H, 4.03; N, 11.19; found: C, 72.02; H, 4.19; N, 11.00 .

2-(5-Methyl-pyridazin-3-yl) naphthalene-1,4-dione (38). Obtained by reaction of naphthalene1,4-dione $7(0.158 \mathrm{~g}, 1 \mathrm{mmol})$ and 4-methyl pyridazine $(0.095 \mathrm{~g}, 1 \mathrm{mmol})$ as a viscous liquid, yield $0.058 \mathrm{~g}, 23 \% ;{ }^{1} \mathrm{H}$ NMR $\left(\mathrm{CDCl}_{3}\right): \delta 8.86(\mathrm{~s}, 1 \mathrm{H}), 8.18(\mathrm{~s}, 1 \mathrm{H}), 8.06(\mathrm{~m}, 2 \mathrm{H}), 7.98(\mathrm{~m}, 2 \mathrm{H})$, $7.50(\mathrm{~s}, 1 \mathrm{H}), 2.40(\mathrm{~s}, 3 \mathrm{H}) ;{ }^{13} \mathrm{C} \mathrm{NMR}\left(\mathrm{CDCl}_{3}\right): \delta 188.2(2 \mathrm{C}), 158.1,154.2,152.4,138.3(2 \mathrm{C})$, 136.6 (2C), 135.4 (2C), 131.2 (2C), 125.2, 22.5; LRMS (ES $\left.{ }^{+}\right): m / z 251.3\left[(\mathrm{M}+\mathrm{H})^{+}, 100 \%\right]$; Anal. Calc for $\mathrm{C}_{15} \mathrm{H}_{10} \mathrm{~N}_{2} \mathrm{O}_{2}$ (250.25): C, 71.99; H, 4.03; N, 11.19; found: C, 71.56; H, 3.98; N, 10.98 .

Acetic acid-6-(1,4-dioxo-1,4-dihydro-naphthalen-2-yl)-pyridin-3-yl ester (39). Obtained by reaction of naphthalene-1,4-dione $7(0.158 \mathrm{~g}, 1 \mathrm{mmol})$ and 3-acetoxy pyridine $(0.138 \mathrm{~g}, 1 \mathrm{mmol})$ as a liquid, yield $0.065 \mathrm{~g}, 22 \% ;{ }^{1} \mathrm{H}$ NMR $\left(\mathrm{CDCl}_{3}\right): \delta 8.86(\mathrm{~s}, 1 \mathrm{H}), 8.78(\mathrm{~s}, 1 \mathrm{H}), 8.06(\mathrm{~m}, 2 \mathrm{H})$, $7.98(\mathrm{~m}, 2 \mathrm{H}), 7.76(\mathrm{~s}, 1 \mathrm{H}), 7.28(\mathrm{~s}, 1 \mathrm{H}), 2.18(\mathrm{~s}, 3 \mathrm{H}) ;{ }^{13} \mathrm{C} \mathrm{NMR}\left(\mathrm{CDCl}_{3}\right): \delta 188.5(2 \mathrm{C}), 170.1$, 158.2, 151.5, 147.0, 145.2, 138.4 (2C), 136.6, 134.6 (2C), 130.6 (2C), 131.0, 122.5, 18.2; LRMS $\left(\mathrm{ES}^{+}\right): m / z 294.4\left[(\mathrm{M}+\mathrm{H})^{+}, 100 \%\right]$; Anal. Calc for $\mathrm{C}_{17} \mathrm{H}_{11} \mathrm{NO}_{4}$ (293.27): C, 69.62; H, 3.78; N, 4.78; found: C, 69.34; H, 3.86; N, 4.23.

2-(5-Acetyl-1-methyl-1H-pyrrol-2-yl) naphthalen-1,4-dione (40). Obtained by reaction of naphthalene-1,4-dione $7(0.158 \mathrm{~g}, 1 \mathrm{mmol})$ and 2-acetyl-1-methyl pyrrole $(0.084 \mathrm{~g}, 1 \mathrm{mmol})$ as a viscous liquid, yield $0.067 \mathrm{~g}, 24 \% ;{ }^{1} \mathrm{H} \mathrm{NMR}\left(\mathrm{CDCl}_{3}\right): \delta 8.18(\mathrm{~s}, 1 \mathrm{H}), 8.06(\mathrm{~m}, 2 \mathrm{H}), 7.98(\mathrm{~m}, 2 \mathrm{H})$, $6.72-6.68(\mathrm{~m}, 2 \mathrm{H}), 3.66(\mathrm{~s}, 3 \mathrm{H}), 2.58(\mathrm{~s}, 3 \mathrm{H}) ;{ }^{13} \mathrm{C} \mathrm{NMR}\left(\mathrm{CDCl}_{3}\right): \delta 188.2(2 \mathrm{C}), 186.6,154.8$, 138.2 (2C), 137.2, 136.7, 135.2 (2C), 131.2 (2C), 130.4, 118.2, 112.3, 26.8, 22.5; $\mathrm{LRMS}_{(\mathrm{ES}}^{+}$): $m / z 280.3\left[(\mathrm{M}+\mathrm{H})^{+}, 100 \%\right]$; Anal. Calc for $\mathrm{C}_{17} \mathrm{H}_{13} \mathrm{NO}_{3}$ (279.29): C, 73.11; H, 4.69; N, 5.02; found: C, 73.28; H, 4.85; N, 5.10.

6-(1,4-Dioxo-1,4-dihydro-naphthalen-2-yl)-pyrazine-2-carboxylic acid methyl ester (41). Obtained by reaction of naphthalene-1,4-dione $7(0.158 \mathrm{~g}, 1 \mathrm{mmol})$ and methyl-2-pyrazine carboxylate $(0.139 \mathrm{~g}, 1 \mathrm{mmol})$ as a viscous liquid, yield $0.094 \mathrm{~g}, 32 \% ;{ }^{1} \mathrm{H} \mathrm{NMR}\left(\mathrm{CDCl}_{3}\right): \delta 8.92$ $(\mathrm{s}, 1 \mathrm{H}), 8.78(\mathrm{~s}, 1 \mathrm{H}), 8.16(\mathrm{~s}, 1 \mathrm{H}), 8.08(\mathrm{~m}, 2 \mathrm{H}), 7.88(\mathrm{~m}, 2 \mathrm{H}), 3.78(\mathrm{~s}, 3 \mathrm{H}) ;{ }^{13} \mathrm{C} \mathrm{NMR}\left(\mathrm{CDCl}_{3}\right): \delta$ 188.3 (2C), 168.5, 154.8, 152.0, 146.7, 145.2, 144.1, 138.7 (2C), 137.2, 135.6 (2C), 131.2 (2C), 52.0; LRMS (ES $\left.{ }^{+}\right): m / z 295.3\left[(\mathrm{M}+\mathrm{H})^{+}, 100 \%\right.$ ]; Anal. Calc for $\mathrm{C}_{16} \mathrm{H}_{10} \mathrm{~N}_{2} \mathrm{O}_{4}$ (294.26): C, 65.31; H, 3.43; N, 9.52; found: C, 65.53; H, 3.55; N, 9.65 . 


\section{Acknowledgements}

Authors are thankful to the Tuberculosis Antimicrobial Acquisition and Coordination Facility (TAACF), which provided antimycobacterial data through a research and development contract with the U.S. National Institute of Allergy and Infectious Diseases.

\section{References}

1. World Health Organization. Global Tuberculosis Control: Surveillance, Planning, Financing. WHO Report 2006; Geneva, Switzerland, ISBN 92-4 156314-1.

2. Snider, D. E.; Raviglione, M.; Kochi, A. Bloom, B., Ed.; ASM: Washington, DC, 1994, p 3.

3. Dye, C.; Scheele, S.; Dolin, P.; Pathania, V.; Raviglione, M. C. J. Am. Med. Assoc. 1999, $282,677$.

4. Sprat, B. G. Science 1994, 264, 360.

5. Ballell, L.; Field, R. A.; Duncan, K.; Young, R. J. Antimicrob. Agents Chemother. 2005, 49, 2153.

6. Duncan, K.; Barry, C. E. Curr. Opin. Microbiol. 2004, 7, 1.

7. Pozniak, A. Ann. N. Y. Acad. Sci. 2001, b, 192.

8. Papageorgiou, V. P.; Assimoponlou, A. N.; Couladouros, E. A.; Hepworth, D.; Nicolaou, K. C. Angew. Chem. Int. Ed. 1999, 38, 270.

9. Osman, S. A. A.; Abdalla, A. A.; Alaib, M. O. J. Pharm.Sci. 1983, 72, 68.

10. Tandon, V. K.; Maurya, H. K.; Yadav, D. B.; Tripathi, A.; Kumar, M.; Shukla, P. K. Bioorg. Med. Chem. Lett. 2006, 16, 5883.

11. Brinkworth, R. I.; Fairlie, D. P. Biochim. Biophys. Acta. 1995, 1253, 5.

12. Salmon-Chemin, L.; Buisine, E.; Yardley, V.; Kohler, S.; Debreu, M. A.; Landry, V.; Sergheraert, C.; Croft, S. L.; Krauth-Siegel, L. R.; Davioud-Charvet, E. J. Med. Chem. 2001, $44,548$.

13. Lee, E. J.; Lee, H. J.; Park, H. J.; Min, H. Y.; Suh, M. E.; Chung, H. J.; Lee, S. K. Bioorg. Med. Chem. Lett. 2004, 14, 5175.

14. Yardley, V.; Snowdon, D.; Croft, S.; Hazra, B. Phytother. Res. 1996, 10, 559.

15. Bullock, F. J.; Tweedie, J. F.; McRitchie, D. D.; McRitchi, D. D.; Tucker, M. A. J. Med. Chem. 1970, 13, 550.

16. Perry, N. B.; Blunt, J. W. J. Nat. Prod. 1991, 54, 978.

17. Georgiadis, M. P.; Couladouros, E. A.; Delitheos, A. K. J. Pharm. Sci. 1992, 81, 1126.

18. Saquib, M.; Gupta, M. K.; Sagar, R.; Prabhakar, T. S.; Shaw, A. K.; Kumar, R.; Maulik, P. R.; Gaikwad, A. N.; Sinha, S.; Srivastava, A. K.; Chaturvedi, V.; Srivastava, R.; Srivastava, B. S. J. Med. Chem. 2007, 50, 2942.

19. Lall, N.; Meyer, J. J. M. J. Ethnopharmacol. 1999, 66, 347.

20. Lall, N.; Meyer, J. J. M. J. Ethnopharmacol. 2001, 78, 213. 
21. Lall, N.; Das Sharma, M.; Hazra, B.; Meyer, J. J. M. J. Antimicrob. Chemother. 2003, 51, 435.

22. Lall, N.; Meyer, J. J. M.; Wang, Y.; Bapela, N. B.; Van Rensburg, C. E. J.; Fourie, B.; Franzblau, S. G. Pharm. Biol. 2005, 43, 353.

23. Chemin, L. S.; Buisine, E.; Yardley, V.; Kohler, S.; Debreu, M. A.; Landry, V.; Sergheraert, C.; Croft, S. L.; Siegel, R. L. K.; Charvet, E. D. J. Med. Chem. 2001, 44, 548.

24. Mital, A.; Negi, V. S.; Ramachandran, U. Arkivoc 2006, (x), 220.

25. Mital, A.; Negi, V. S.; Ramachandran, U. Accepted in Medicinal Chemistry 2008, In press.

26. Itahara, T.; J. Org. Chem. 1985, 50, 5546.

27. Franzblau, S. G.; Witzig, R. S.; McLaughlin, J. C.; Torres, P.; Madico, G.; Hernandez, A.; Degnan, M. T.; Cook, M. B.; Quenzer, V. K.; Ferguson, R. M.; Gilman, R. H. J. Clin. Microb. 1998, 36, 362.

28. Collins, L.; Franzblau, S. G. Antimicrob. Agents Chemother. 1997, 41, 1004. 